\title{
THE DIFFUSION LIMIT OF TRANSPORT EQUATIONS DERIVED FROM VELOCITY-JUMP PROCESSES*
}

\author{
THOMAS HILLEN ${ }^{\dagger}$ AND HANS G. OTHMER ${ }^{\ddagger}$
}

\begin{abstract}
In this paper we study the diffusion approximation to a transport equation that describes the motion of individuals whose velocity changes are governed by a Poisson process. We show that under an appropriate scaling of space and time the asymptotic behavior of solutions of such equations can be approximated by the solution of a diffusion equation obtained via a regular perturbation expansion. In general the resulting diffusion tensor is anisotropic, and we give necessary and sufficient conditions under which it is isotropic. We also give a method to construct approximations of arbitrary high order for large times. In a second paper (Part II) we use this approach to systematically derive the limiting equation under a variety of external biases imposed on the motion. Depending on the strength of the bias, it may lead to an anisotropic diffusion equation, to a drift term in the flux, or to both. Our analysis generalizes and simplifies previous derivations that lead to the classical Patlak-Keller-Segel-Alt model for chemotaxis.
\end{abstract}

Key words. aggregation, chemotaxis equations, diffusion approximation, velocity-jump processes, transport equations

AMS subject classifications. 35Q80, 60J15, 65U05, 92B05, 35B05, 35K50, 35K55

\section{PII. S0036139999358167}

1. Introduction. There are two major approaches used to describe the motion of biological organisms: (i) a space-jump process in which the individual jumps between sites on a lattice, and (ii) a velocity-jump process in which discontinuous changes in the speed or direction of an individual are generated by a Poisson process [36]. The former leads to a renewal equation in which the kernel governs the waiting time between jumps and the redistribution after a jump and determines the type of partial differential equation that describes the asymptotic behavior of the evolution [36]. In this paper we analyze the diffusion approximation to the transport equation

$$
\frac{\partial}{\partial t} p(x, v, t)+v \cdot \nabla p(x, v, t)=-\lambda p(x, v, t)+\lambda \int_{V} T\left(v, v^{\prime}\right) p\left(x, v^{\prime}, t\right) d v^{\prime}
$$

describing a velocity-jump process. Here $p(x, v, t)$ denotes the density of particles at spatial position $x \in \Omega \subset \mathbb{R}^{n}$, moving with velocity $v \in V \subset \mathbb{R}^{n}$ at time $t \geq 0$ [36]. Here $\lambda$ is the (constant) turning rate and $1 / \lambda$ is a measure of the mean run length between velocity jumps. In general $\lambda$ may be space dependent and depend on internal and external variables as well. The turning kernel $T\left(v, v^{\prime}\right)$ gives the probability of a velocity jump from $v^{\prime}$ to $v$ if a jump occurs, and implicit in the above formulation is the assumption that the choice of a new velocity is independent of the run length. The turning kernel may also be space dependent. When applied to the bacterium $E$. coli, the kernel $T$ includes a bias, as described later, and the turning frequency must

*Received by the editors June 23, 1999; accepted for publication (in revised form) March 29, 2000; published electronically August 29, 2000.

http://www.siam.org/journals/siap/61-3/35816.html

${ }^{\dagger}$ Department of Mathematics, University of Utah, Salt Lake City, UT 84112 (hillen@math.utah. edu). This research of this author was supported by the Deutsche Forschungsgemeinschaft.

$¥$ Department of Mathematics, University of Minnesota, Minneapolis, MN 55455 (ohmer@math. umn.edu). The research of this author was supported in part by NIH grant GM 29123 and by NSF grant DMS 9805494. 
depend on the extracellular signal, as transduced through the signal transduction and motor control system. When applied to the amoeboid cell Dictyostelium discoideum (Dd), which uses both run length control and taxis [13], both the turning kernel and the turning rate must depend indirectly on the extracellular cyclic adenosine monophosphate distribution. In Part II we show how these can be described by including dependence on the velocity and external stimuli in the kernel and/or run length [37].

The backward equation that corresponds to (1.1) has been derived from the underlying stochastic velocity-jump process by Stroock [45] to describe the motion of bacteria. It has also been derived and analyzed in a more general framework by Papanicolaou [39], and we compare our results with his in detail in the discussion section. We do not derive (1.1) rigorously as the forward equation of the velocity-jump process, but instead take it as the starting point of our analysis. From the mathematical point of view the transport equation (1.1) is similar to the Boltzmann equation, in which the right-hand side is an integral operator that describes collision of two particles, and is therefore quadratic in $p$ [4]. The kernel of the integral operator is specified by the dynamics, and it is known that an appropriate scaling of space and time leads at least formally to a diffusion process $[30,17]$. As we show here, this also holds for velocityjump processes as described by (1.1) for one particle and for more general transport processes which also include acceleration terms and other forces (see also Hersh [22], Papanicolaou [39], or Patlak [40]). Alt [1] has used Patlak's approach to relate the chemotactic velocity to the gradient of an attractant or repellent. Alt's derivation, which will be discussed in detail in Part II, is based on a very specific model for the motion of crawling cells, and a number of assumptions used in the derivation make it difficult to interpret the equations in other contexts.

The simplest example that illustrates the regime in which a diffusion equation can be obtained from a velocity-jump process arises in one space dimension. Here a particle moves along the $x$-axis at a speed s, taken to be constant for simplicity, and at random instants of time it reverses direction according to a Poisson process with constant intensity $\lambda$. Let $p^{ \pm}(x, t)$ be the density of particles that are at $(x, t)$ and are moving to the right $(+)$ and left $(-)$. Then $p^{ \pm}(x, t)$ satisfy the equations

$$
\begin{aligned}
& \frac{\partial p^{+}}{\partial t}+s \frac{\partial p^{+}}{\partial x}=-\lambda p^{+}+\lambda p^{-}, \\
& \frac{\partial p^{-}}{\partial t}-s \frac{\partial p^{-}}{\partial x}=\lambda p^{+}-\lambda p^{-} .
\end{aligned}
$$

The density of particles at $(x, t)$ is $p(x, t) \equiv p^{+}(x, t)+p^{-}(x, t)$, and the particle flux is $j \equiv s\left(p^{+}-p^{-}\right)$. These satisfy the equations

$$
\begin{aligned}
& \frac{\partial p}{\partial t}+\frac{\partial j}{\partial x}=0 \\
& \frac{\partial j}{\partial t}+2 \lambda j=-s^{2} \frac{\partial p}{\partial x}
\end{aligned}
$$

and the initial conditions $p(x, 0)=p_{0}(x), j(x, 0)=j_{0}(x)$, where $p_{0}$ and $j_{0}$ are determined from the initial distribution of $p^{+}$and $p^{-}$. This system leads to the telegraph equation

$$
\frac{\partial^{2} p}{\partial t^{2}}+2 \lambda \frac{\partial p}{\partial t}=s^{2} \frac{\partial^{2} p}{\partial x^{2}}
$$


and the diffusion equation results formally in the limit $\lambda \rightarrow \infty, s \rightarrow \infty$ with $s^{2} / \lambda \equiv$ $D$ constant. A rigorous justification of this limit can be obtained directly from an asymptotic analysis of the exact solution or from the more general result stated in Theorem 4.1.

This process was studied by Taylor [47], Fürth [14], Goldstein [15], and subsequently by Kac [28], McKean [32], Segel [43], and Othmer, Dunbar, and Alt [36]. In Part II and in [26] it is shown that if the speed depends on the signal, then cells accumulate at the minima of the speed distribution. If the turning rate depends on the signal distribution, then they can aggregate if the turning rate is not constant and if it also depends on the direction of motion. However, the reduction of the general velocity-jump process to a telegraph equation is possible only in one space dimension; even with two dimensions and four velocities the system does not reduce to a telegraph equation in any scaling. However, an alternate approach to transport based on a theory of mixtures leads to the telegraph equation in any number of dimensions [35], and these two different approaches have not been reconciled.

Nonlinear versions of the one-dimensional telegraph process, which also include birth, death, and interactions of particles, have been studied by Dunbar and Othmer [9], Dunbar [8], Holmes [27], Hadeler [18, 19, 20], Hillen [23, 24, 25, 26], Müller and Hillen [34], and Schneider and Müller [42]. Asymptotic estimates for classical solutions have been derived and the upper semicontinuity of the attractor has been proven [42].

In the following section we state the general assumptions on the turning kernel $T$ which ensure that the turning operator defined there is positive in an appropriate sense (see Definition 2.1). The positivity in turn guarantees that a diffusion limit of the jump process exists. In section 3 we introduce the parabolic scaling and formally derive the parabolic limit equation. Since the parabolic limit is the outer solution in singular perturbations terms, these higher approximations depend only on the initial values for the parabolic limit problem, which is also true for the Chapman-EnskogHilbert expansion of Boltzmann's equation, and which is known as Hilbert's paradox in that context $[12,30,17]$. We also derive several equivalent conditions on the turn angle distribution under which the diffusion matrix is a scalar multiple of the identity. It turns out that the diffusion constant depends on the second eigenvalue of the turning operator defined later (cf. Theorem 3.5). In section 4 we first study the limit equation itself and then use general properties of such equations to prove the approximation result stated in Theorem 4.1. In the discussion section we compare our approach and results with a number of previous analyses of this problem. We also briefly describe the important aspects of the application of this approach to chemotaxis, which are developed fully in Part II. In Part II we systematically analyze signaldependent turning rates and redistribution kernels. We give an example of an order one, anisotropic perturbation of the redistribution kernel that nonetheless leads to a scalar (hence isotropic) diffusion matrix. We also analyze $\mathcal{O}(\epsilon)$ perturbations of the turning kernel and turning rate and show how the chemotactic velocity and sensitivity are obtained from more fundamental and measurable properties of the motion. This leads to a variety of different types of signal dependence of turning rates and kernels for which the jump process is asymptotically described by the Patlak-Keller-Segel-Alt (PKSA) equation.

\section{The mathematical setup.}

2.1. Properties of the turning operator. In the following we deal primarily with the case $\Omega=\mathbb{R}^{n}$, since our interest lies in understanding how the properties of the turning kernel are reflected in the parabolic limit equations. Boundary conditions for 
transport equations of the form (1.1) in bounded domains have been studied previously [3], and it is known which conditions are sufficient to produce Neumann boundary conditions in the diffusion approximation. We also suppose that the velocities lie in a compact set $V \subset \mathbb{R}^{n}$ and that $V$ is symmetric with respect to the origin, which is no restriction for the applications. In many applications it is assumed that the kernel $T$ is symmetric or that it is continuous (see, e.g., [1]). While the symmetry condition may be appropriate in a homogeneous, isotropic environment, we can relax this condition with very little effort and still obtain a parabolic equation in the diffusion limit.

Let $\mathcal{K}$ denote the cone of nonnegative functions in $L^{2}(V)$, and for fixed $(x, t)$ define an integral operator $\mathcal{T}$ by

$$
\mathcal{T} p=\int_{V} T\left(v, v^{\prime}\right) p\left(x, v^{\prime}, t\right) d v^{\prime}
$$

Its adjoint is given by

$$
\mathcal{T}^{*} p=\int_{V} T\left(v^{\prime}, v\right) p\left(x, v^{\prime}, t\right) d v^{\prime} .
$$

We make the following assumptions about the kernel and the integral operator.

(T1) $T\left(v, v^{\prime}\right) \geq 0, \quad \int_{V} T\left(v, v^{\prime}\right) d v=1, \quad$ and $\quad \int_{V} \int_{V} T^{2}\left(v, v^{\prime}\right) d v^{\prime} d v<\infty$.

(T2) There are functions $u_{0}, \phi$, and $\psi \in \mathcal{K}$ with the properties that $u_{0} \not \equiv 0$ and $\phi$ and $\psi$ vanish at most on a set of Lebesgue measure zero, and such that for all $\left(v, v^{\prime}\right) \in V \times V$

$$
u_{0}(v) \phi\left(v^{\prime}\right) \leq T\left(v^{\prime}, v\right) \leq u_{0}(v) \psi\left(v^{\prime}\right)
$$

(T3) $\|\mathcal{T}\|_{\langle 1\rangle}<1$, where $\langle 1\rangle^{\perp}$ is the orthogonal complement in $L^{2}(V)$ of the span of 1 .

(T4) $\int_{V} T\left(v, v^{\prime}\right) d v^{\prime}=1$.

The first two conditions in (T1) imply that $T\left(\cdot, v^{\prime}\right)$ is a nonnegative probability distribution on $V$, and together with the third condition in (T1) guarantee that $\mathcal{T}$ and its adjoint $\mathcal{T}^{*}$ both map $\mathcal{K} \rightarrow \mathcal{K}$. We will use condition (T2) to show that $\mathcal{T}^{*}$ is $u_{0}$-positive, as defined below, and as a result, we can show that $\mathcal{T}^{*}$ has a simple dominant eigenvalue equal to one with a corresponding positive eigenfunction identically constant. Finally, we use condition (T3) to show that the limiting equation indeed is of parabolic type (Lemma 3.3).

It follows from (T1) that for every fixed $(x, t)$, both $\mathcal{T}$ and its adjoint are HilbertSchmidt operators (hence bounded and compact) on $L^{2}(V)$ (see $[10,21]$ for general definitions). Therefore both $\mathcal{T}$ and $\mathcal{T}^{*}$ have a pure point spectrum and their nonzero eigenvalues have finite multiplicity. However, more can be said, since $\mathcal{T}$ and $\mathcal{T}^{*}$ map $\mathcal{K}$ into itself. To make this precise we need the following definitions.

DEFINITION 2.1.

1. Let $X$ be a Banach space and $K$ the positive cone in $X$. Let $L: X \longrightarrow X$; then $L$ is positive if $L: K \longrightarrow K$, i.e., $\phi \in K ; \phi \geq 0$ implies that $L \phi \geq 0$.

2. Let $L$ be positive. Then $L$ is $u_{0}$-bounded from below (above) if there is a fixed $u_{0} \in K, u_{0} \neq 0$ such that for every $\phi \in K$ there exists an $n$ and $\alpha>0(m$ and $\beta>0)$ such that

$$
\alpha u_{0} \leq L^{n} \phi, \quad\left(L^{m} \phi \leq \beta u_{0}\right) .
$$

If $L$ is $u_{0}$-bounded above and below then $L$ is $u_{0}$-positive. 
3. $K$ is reproducing if for all $\phi \in X$ there exist $u, v \in K$ such that $\phi=u-v$.

The following theorem gives the general Perron-Frobenius property of $u_{0}$-positive operators that is needed later.

Theorem 2.2 (see Krasnosel'skii [29]). Let $K$ be the nonnegative cone in $X$, let $K$ be reproducing, and let $L$ be $u_{0}$-positive. Let $\varphi_{0} \in K$ and suppose that $\varphi_{0}$ is an eigenfunction of $L$. Then

(i) if $L \varphi_{0}=\mu_{0} \varphi_{0}$, then $\mu_{0}$ is a simple eigenvalue;

(ii) $\varphi_{0}$ is a unique eigenfunction in $K$ to within a scalar factor;

(iii) $\left|\mu_{0}\right|$ is greater than the magnitude of the remaining eigenvalues.

The cone $\mathcal{K}$ is reproducing, because for any $\phi \in L^{2}(V)$ we can write $\phi(v)=$ $\phi^{+}(v)-\phi^{-}(v)$ where

$$
\phi^{+}(v)=\left\{\begin{array}{ll}
\phi(v) & \text { if } \phi(v) \geq 0, \\
0 & \text { otherwise, }
\end{array} \quad \phi^{-}(v)= \begin{cases}0 & \text { if } \phi(v) \geq 0, \\
-\phi(v) & \text { if } \phi(v)<0 .\end{cases}\right.
$$

If $T$ is continuous on a compact set it has a maximum and minimum there, and therefore we can find a nonnegative function $u_{0}$ and a sufficiently large constant $\gamma>0$ such that (2.3) holds with $\phi=1$ and $\psi=\gamma$. In general we have the following result.

Lemma 2.3 (see Krasnosel'skii [29]). If T(·, $)$ satisfies (T1) and (T2), then $\mathcal{T}^{*}$ is $u_{0}$-positive.

We can apply Theorem 2.2 to the adjoint $\mathcal{T}^{*}$ as follows. The preceding lemma and (T1) show that $\mathcal{T}^{*}$ is $u_{0}$-positive and has an eigenvalue equal to 1 . Therefore the theorem shows that $\phi \equiv 1$ is the unique (to within a positive scalar factor) positive eigenfunction and that all other eigenvalues are less than 1 in magnitude. The operators $\mathcal{T}$ and $\mathcal{T}^{*}$ have the same spectral radius which is 1 . Assumption (T4) implies that the operator $\mathcal{T}$ has a positive eigenfunction corresponding to the eigenvalue 1 and that the corresponding eigenfunction can be chosen as $\phi \equiv 1$. The second condition of (T1) implies that the forward jump process conserves particles, and (T4) implies that the same is true for the adjoint evolution equation.

Remark 2.1. It is clear that we could allow $\lambda$ and or $T$ to depend on $x$ and/or $t$ parametrically and the same conclusion would hold pointwise in $x$ and $t$.

To simplify the notation later we define the turning operator

$$
\mathcal{L} p(v)=-\lambda p(v)+\lambda \mathcal{T} p(v)
$$

and its adjoint

$$
\mathcal{L}^{*} p(v)=-\lambda p(v)+\lambda \mathcal{T}^{*} p(v) .
$$

Then the foregoing can be restated in terms of the spectrum of $\mathcal{L}^{*}$ as follows: zero is a simple eigenvalue of $\mathcal{L}^{*}$ with a unique positive eigenfunction $\phi(v)=1$, and all other eigenvalues $\mu$ have $-2 \lambda<\operatorname{Re} \mu<0 .^{1}$

Remark 2.2. Since $\mathcal{T}$ is real and compact, $\mathcal{L}$ and $\mathcal{L}^{*}$ have the same spectrum [46], and it follows that zero is a simple eigenvalue of $\mathcal{L}$. This fact is essential for the perturbation analysis done later.

If the kernel is symmetric $\mathcal{L}$ has a complete orthogonal set of eigenfunctions [46], even if $\mathcal{T}$ has finite rank (in that case $\mathcal{T}$ has an infinite dimensional null space and we can take any orthonormal basis of $\mathcal{N}(\mathcal{T})$ as a complement to $\mathcal{R}(\mathcal{T})$ ). For the

\footnotetext{
${ }^{1}$ As we shall see in the following section, one cannot obtain diffusion equations in the time and space scalings used here unless $\mathcal{L}$ has a $v$-independent eigenfunction corresponding to the zero eigenvalue, and thus the conservation property for both forward and reverse processes is essential.
} 
general theory developed here we do not assume that the kernel is symmetric, but we may have a complete set nonetheless. Since $\mathcal{T}$ is a Hilbert-Schmidt operator, general conditions are known under which the eigenfunctions are complete (cf. [10, p. 1099, et seq.])

The second condition in assumption (T1) implies that any eigenfunction of $\mathcal{L}$ corresponding to a nonzero eigenvalue has mean zero over $V$, and therefore $L^{2}(V)=$ $\langle 1\rangle \oplus\langle 1\rangle^{\perp}$. Condition (T3) implies that for all $\psi \in\langle 1\rangle^{\perp}$

$$
\int \psi \mathcal{L} \psi d v \leq-\mu_{2}\|\psi\|_{L^{2}(V)}^{2}
$$

where

$$
\mu_{2} \equiv \lambda\left(1-\|\mathcal{T}\|_{\langle 1\rangle \perp}\right)
$$

We collect all the above properties together in the following theorem.

Theorem 2.4. Assume (T1)-(T4); then

1. 0 is a simple eigenvalue of $\mathcal{L}$ and the corresponding eigenfunction is $\phi(v) \equiv 1$.

2. All nonzero eigenvalues satisfy $-2 \lambda<R e \mu \leq-\mu_{2}<0$, and to within a scalar factor there is no other positive eigenfunction.

3. There is a decomposition $L^{2}(V)=\langle 1\rangle \oplus\langle 1\rangle^{\perp}$ and estimate (2.4) holds.

4. $\|\mathcal{L}\|_{\mathcal{L}\left(L^{2}(V), L^{2}(V)\right)} \leq 2 \lambda$.

5. $\mathcal{L}$ restricted to $\langle 1\rangle^{\perp} \subset L^{2}(V)$ has a linear inverse $\mathcal{F}$ with norm

$$
\|\mathcal{F}\|_{\mathcal{L}\left(\langle 1\rangle^{\perp},\langle 1\rangle^{\perp}\right)} \leq \frac{1}{\mu_{2}} .
$$

If $\mathcal{T}$ is normal in addition to being compact, which requires that

$$
\int T\left(v, v^{\prime \prime}\right) T\left(v^{\prime}, v^{\prime \prime}\right) d v^{\prime \prime}=\int T\left(v^{\prime \prime}, v\right) T\left(v^{\prime \prime}, v^{\prime}\right) d v^{\prime \prime} \quad \text { for all }\left(v, v^{\prime}\right) \in V \times V
$$

then $\mathcal{L}$ has a complete set of eigenfunctions since $I-\mathcal{T}$ is normal and has a pure point spectrum. As a result there is an orthogonal decomposition of $L^{2}(V)$ into eigenspaces of $\mathcal{L}$ (cf. [6]). In this case (T3) is unnecessary, and $\mathcal{L}$ has the spectral representation given in the following theorem.

Theorem 2.5 (cf. Conway [6, p. 55 et seq.]). Assume that condition (T1) holds and that $\mathcal{T}$ is normal. Then $\mathcal{L}$ has a countable number of distinct eigenvalues $\left\{0, \mu_{2}, \mu_{3}, \ldots\right\}$ with $\mu_{j} \neq 0$ for $j \geq 2$. Associated with each $\mu_{j}$ there is a spectral projection $P_{j}: V \rightarrow V$ such that $\mathcal{L}$ has the representation

$$
\mathcal{L}=\sum_{j=2}^{\infty} \mu_{j} P_{j}
$$

As we will see in the following section, it is particularly simple to evaluate certain solvability conditions that arise in the perturbation analysis when such a spectral representation exists.

2.2. The shift operator $\boldsymbol{A}=-\boldsymbol{v} \cdot \nabla$. The shift operator $A=-(v \cdot \nabla)$ with domain

$$
\mathcal{D}(A)=\left\{\phi \in L^{2}\left(\mathbb{R}^{n} \times V\right): \phi(., v) \in H^{1}\left(\mathbb{R}^{n}\right)\right\}
$$


is skew adjoint in $L^{2}\left(\mathbb{R}^{n} \times V\right)$. Hence it is dissipative in the sense of Pazy [41]. Then the linear transport equation defines a strongly continuous semigroup on $L^{2}\left(\mathbb{R}^{n} \times V\right)$. Since the turning operator $\mathcal{L}$ is linear and bounded we have the following existence result (see [41, Chapter 3, Theorem 1.1]).

Theorem 2.6. Assume (T1). For each initial condition $p_{0} \in \mathcal{D}(A)$ there exists a unique solution of the transport equation (1.1) in

$$
\mathcal{X}=C^{1}\left([0, \infty), L^{2}\left(\mathbb{R}^{n} \times V\right)\right) \cap C([0, \infty), \mathcal{D}(A)) .
$$

3. The formal diffusion approximation. One can only expect to obtain a diffusion process as an asymptotic description of the velocity-jump process if there are time and space scales on which there are many velocity jumps in order one time, but a small net displacement on this time scale. For the purpose of identifying these scales, we assume that $\lambda$ and $T$ are independent of the spatial position and that $\lambda$ is independent of velocity. To motivate the appropriate scaling of space and time, we first identify a length scale $L$ that is characteristic of the macroscopic evolution. For instance, this may be the size of the domain on which an experiment is done, or, if there is an external field $S$ to which the organisms respond, one may choose

$$
L=\max \frac{S}{|\nabla S|},
$$

where the maximum is taken over $\bar{\Omega}$ and a suitable compact time interval. Other functionals of this ratio, as well as other characteristic lengths, may be appropriate, but whatever the choice we assume that $L$ is a fixed constant.

Define the dimensionless velocity, space, and time variables

$$
u=\frac{v}{s}, \quad \xi=\frac{x}{L}, \quad \tau=\frac{t}{\sigma},
$$

where $s$ is a characteristic speed and $\sigma$ is as yet undetermined. Then (1.1) can be written

$$
\frac{1}{\sigma} \frac{\partial \tilde{p}}{\partial \tau}+\left(\frac{s}{L}\right) u \cdot \nabla_{\xi} \tilde{p}=-\lambda \tilde{p}+\lambda \int T\left(u, u^{\prime}\right) \tilde{p}\left(\xi, u^{\prime}, \tau\right) d u^{\prime},
$$

where $\nabla_{\xi}$ is the gradient in $\xi$ coordinates, and $\tilde{p}(\xi, u, \tau) \equiv p(\xi L, u s, \sigma \tau)$. We have used the fact that $T$ is a probability kernel in applying the coordinate change.

We can estimate a diffusion coefficient as the product of the characteristic speed times the average distance traveled between velocity jumps, which gives $D \sim \mathcal{O}\left(s^{2} / \lambda\right)$, as in the example given in the introduction. From this we obtain the characteristic diffusion time for diffusion on the length scale L (i.e., $\xi \sim \mathcal{O}(1)$ ) as

$$
\tau_{D I F F} \sim \frac{L^{2}}{D}=\frac{L^{2} \lambda}{s^{2}} .
$$

We can also define a characteristic drift time as

$$
\tau_{D R I F T}=\frac{L}{s},
$$

and we assume that the space scale $L$ is such that the time scales are related as follows:

$$
\tau_{R U N} \equiv \lambda^{-1} \ll \tau_{D R I F T} \ll \tau_{D I F F} .
$$


For example, a characteristic speed for bacteria such as E. coli is $10-20 \mu / \mathrm{sec}$, and $\lambda^{-1} \sim \mathcal{O}(1)$ second. On a length scale of $1 \mathrm{~mm}, \tau_{D R I F T} \sim 50-100$ seconds and $\tau_{D I F F} \sim 2500-10^{4}$ seconds. Therefore we have $\tau_{R U N} \sim \mathcal{O}(1)$ on the dimensional scale, and

$$
\begin{aligned}
\tau_{D R I F T} & \sim O(1 / \epsilon), \\
\tau_{D I F F} & \sim O\left(1 / \epsilon^{2}\right),
\end{aligned}
$$

where $\epsilon \sim \mathcal{O}\left(10^{-2}\right)$. We can ensure that this hierarchy holds in general by assuming that the time scale is such that $\lambda \sim \mathcal{O}(1)$ and that $L$ is chosen so the $L \sim \mathcal{O}(s / \varepsilon)$.

If we now set $\sigma=\tau_{D I F F}$, use the fact that $\tau_{D R I F T} \sim \mathcal{O}(1 / \epsilon)$, and revert to $v$ for the scaled velocity, we can write (3.1) as

$$
\varepsilon^{2} \frac{\partial p}{\partial \tau}+\varepsilon v \cdot \nabla p=-\lambda p+\lambda \int_{V} T\left(v, v^{\prime}\right) p\left(\xi, v^{\prime}, \tau\right) d v^{\prime}
$$

with $\tau=\varepsilon^{2} \mathrm{t}, \xi=\varepsilon x / s, v \in V$, and $\xi \in \tilde{\Omega}$ with $\tilde{\Omega}=\varepsilon \Omega / s$. Here and hereafter we drop the subscript on $\nabla$ and the tilde on $p$. In view of the space and time scalings chosen, we assume that $\lambda \sim \mathcal{O}(1)$. Since $\int T\left(v, v^{\prime}\right) d v=1$, it follows that the right-hand side of (3.3) is $\mathcal{O}(1)$ compared with the left-hand side, whatever the magnitude of $p$. As we show next, this leads to a diffusion equation for the lowest order term of an outer expansion.

Since we are only interested in the solution of (3.3) on the diffusion time scale, we assume the regular perturbation expansion

$$
p(\xi, v, \tau)=\sum_{i=0}^{k} p_{i}(\xi, v, \tau) \varepsilon^{i}+\varepsilon^{k+1} p_{k+1}(\xi, v, \tau) .
$$

This ansatz gives the "outer" solution in the sense of singular perturbations and is similar to what is used in the context of the Boltzmann equation, where it is called a Hilbert expansion (see, e.g., $[12,30,17]$ ). Comparing terms of equal order in $\varepsilon$, we obtain the following system of equations:

$$
\begin{array}{rlrl}
\varepsilon^{0}: & \mathcal{L} p_{0} & \equiv-\lambda p_{0}+\lambda \int_{V} T\left(v, v^{\prime}\right) p_{0}\left(\xi, v^{\prime}, \tau\right) d v^{\prime}=0, \\
\varepsilon^{1}: & \mathcal{L} p_{1} & =v \cdot \nabla p_{0}, \\
\varepsilon^{2}: & \mathcal{L} p_{2} & =\frac{\partial p_{0}}{\partial \tau}+v \cdot \nabla p_{1}, \\
\vdots & \\
\varepsilon^{i}: & \mathcal{L} p_{i}=\frac{\partial p_{i-2}}{\partial \tau}+v \cdot \nabla p_{i-1}, \quad 3 \leq i \leq k+1 .
\end{array}
$$

The first equation and (T4) imply that $p_{0}$ is independent of $v$. Since $\mathcal{L}$ is singular the right-hand side of (3.6) must satisfy a solvability condition. The eigenfunction corresponding to the eigenvalue $\mu=0$ of $\mathcal{L}^{*}$ is $\phi_{0} \equiv 1$, and therefore this condition is

$$
\int_{V}\left(v \cdot \nabla p_{0}\right) d v=0
$$

which can be written alternatively as

$$
\nabla \cdot j_{0}=0
$$


where the flux $j_{i}$ of $p_{i}$ for $i=1, \ldots, k+1$ is defined as

$$
j_{i}=\int_{V} v p_{i} d v
$$

Since $V$ is symmetric and $p_{0}$ is independent of $v,(3.9)$ is satisfied.

It remains to solve (3.6) subject to (3.9) or (3.10). Since the total number of particles is conserved, the solution must satisfy

$$
\int_{\tilde{\Omega}} \int_{V} p(\xi, v, \tau) d \xi d v=\int_{\tilde{\Omega}} \int_{V}\left(p_{0}(\xi, v, \tau)+\varepsilon p_{1}(\xi, v, \tau)+\cdots\right) d \xi d v=N_{0},
$$

where $N_{0}$ is a constant. We can stipulate that all the mass is in $p_{0}$, and that

$$
\int_{V} p_{j} d v=0
$$

for $j \geq 1$. Since $\mathcal{L}$ is a Fredholm operator, (3.6) can be solved via a generalized or pseudoinverse [46]. Then the solution can be written

$$
p_{1}=\mathcal{F}\left(v \cdot \nabla p_{0}\right),
$$

where

$$
\mathcal{F}=\left(\left.\mathcal{L}\right|_{\langle 1\rangle^{\perp}}\right)^{-1}
$$

is a linear functional on $\langle 1\rangle^{\perp} \subset L^{2}(V)$ defined by the pseudoinverse of $\mathcal{L}$.

Fortunately it is possible to calculate the pseudoinverse $\mathcal{F}$ explicitly in many cases. For example, if $\mathcal{L}$ is normal the construction of the inverse reduces to a computation of eigenvalues and eigenvectors, since $\mathcal{F}$ has a spectral representation in that case.

Lemma 3.1. Under the conditions of Theorem 2.5 the inverse $\mathcal{F}$ of $\mathcal{L}$ on $\langle 1\rangle^{\perp}$ has the representation

$$
\mathcal{F}=\sum_{j=2}^{\infty} \frac{P_{j}}{\mu_{j}} .
$$

The evolution equation for $p_{0}$ is obtained from the solvability condition at $O\left(\varepsilon^{2}\right)$, which is given by

$$
\int_{V}\left[\frac{\partial p_{0}}{\partial \tau}+v \cdot \nabla\left(\mathcal{F}\left(v \cdot \nabla p_{0}\right)\right)\right] d v=0
$$

Since $p_{0}$ is independent of $v$ we can write this as

$$
\frac{\partial p_{0}}{\partial \tau}-\nabla \cdot\left(D \nabla p_{0}\right)=0
$$

where the diffusion tensor is defined as

$$
D \equiv-\frac{1}{\omega} \int_{V} v \mathcal{F} v d v
$$


Is is also clear from the definition (3.16) that $D$ is symmetric if $\mathcal{F}$ acts on $v$ by scalar multiplication, and the positivity of $D$ follows from the positivity of $\mathcal{T}$ and the location of its spectrum. This is shown in Lemma 3.3.

Remark 3.1. If we allow $\lambda$ and/or $T$ to depend on $x$ and/or $t$, then the pseudoinverse does also and the diffusion tensor and the chemotactic velocity defined later may depend on $x$ and/or $t$ as well. We can also allow a space-dependent speed if the jump process only changes the direction of the particle.

Example 3.1. Suppose that $V=s S^{n-1}$ and $T\left(v, v^{\prime}\right)=1 / \omega, \omega=|V|$, i.e., that the redistribution is uniform in velocity space, and that $\lambda$ is a constant. One finds that the pseudoinverse $\mathcal{F}$ for this kernel is simply multiplication by $-\lambda^{-1}$, and therefore, under the condition (3.12) we have

$$
p_{1}=-\frac{1}{\lambda}\left(v \cdot \nabla p_{0}\right)
$$

As a check we note that

$$
\int_{V} p_{1} d v=-\frac{1}{\lambda} \nabla \cdot j_{0}=0
$$

by (3.10), and we find that the diffusion tensor is given by

$$
D=\frac{1}{\omega} \int_{V} \frac{v v}{\lambda} d v=\frac{s^{2}}{\lambda n} I
$$

which is clearly positive definite and isotropic. ${ }^{2}$

3.1. The parabolic limit equation. We next prove that the initial value problem

$$
\begin{aligned}
& \frac{\partial p_{0}}{\partial \tau}-\nabla \cdot\left(D \nabla p_{0}\right)=0 \\
& p_{0}(\xi, 0)=\int_{V} p(\xi, v, 0) d v
\end{aligned}
$$

is genuinely parabolic for any $D$ defined by (3.16) if (T1)-(T4) hold true.

TheOREM 3.2. Assume (T1)-(T4) and let $p(., v, 0) \in L^{2}(\tilde{\Omega})$ for each $v \in V$. Then there exists a unique global solution $p_{0}$ of (3.18) with the following properties:

$$
\begin{aligned}
& \text { (i) } p_{0} \in C\left([0, \infty), L^{2}(\tilde{\Omega})\right), \\
& \text { (ii) } \frac{\partial p_{0}}{\partial \tau} \in C^{\infty}(\tilde{\Omega}) \quad \text { for each } \tau>0, \\
& \text { (iii) }\left\|p_{0}(., \tau)\right\|_{C^{\infty}} \quad \text { is decreasing as a function of } \tau \text {. }
\end{aligned}
$$

Proof. We show that the differential operator in (3.18) is strictly elliptic on $L^{2}(\tilde{\Omega})$. Then it generates a contractive analytic semigroup and the above statements follow. This is immediate, using (3.17), when $T\left(v, v^{\prime}\right)=1 / \omega$, since then solutions $p_{0}$ of this equation exist globally and are smooth for each $\tau>0$.

In general the operator $\nabla \cdot D \nabla$ defines an unbounded differential operator on $H^{2}(\tilde{\Omega})$, and it generates an analytic semigroup if the quadratic form generated by the matrix $D: \mathbb{R}^{n} \rightarrow \mathbb{R}^{n}$ is coercive.

\footnotetext{
${ }^{2} \mathrm{By}$ isotropic we mean as usual that $D$ is invariant under all orthogonal transformations. It is well known that the only isotropic second-rank tensor is a multiple of the unit tensor [5].
} 
Lemma 3.3. There exists a positive constant $\kappa>0$ such that

$$
\varphi \cdot D \varphi \geq \kappa|\varphi|^{2}
$$

for each $\varphi \in \mathbb{R}^{n}$.

Proof of Lemma 3.3. For $\varphi \in \mathbb{R}^{n}$ we consider the quadratic form

$$
\varphi \cdot D \varphi=-\frac{1}{\omega} \int_{V}(\varphi \cdot v) \mathcal{F}(\varphi \cdot v) d v .
$$

The term $(\varphi \cdot v)$ is not constant as a function of $v \in V$, hence we can apply $\mathcal{F}$. There is a nonconstant function $z(v) \in\langle 1\rangle^{\perp}$ such that $\mathcal{L} z(v)=\varphi \cdot v$. Then

$$
\varphi \cdot D \varphi=-\frac{1}{\omega} \int_{V} \mathcal{L} z(v) z(v) d v
$$

From (2.4) it follows that

$$
\int \mathcal{L} z(v) z(v) d v \leq-\mu_{2}\|z\|_{2}^{2}<0 .
$$

Then

$$
\varphi \cdot D \varphi \geq \frac{\mu_{2}}{\omega} \int_{V}|z(v)|^{2} d v=\frac{\mu_{2}}{\omega}|\varphi|^{2} \int_{V}\left|\mathcal{F}\left(\frac{\varphi}{|\varphi|} \cdot v\right)\right|^{2} d v \geq \frac{c_{0} \mu_{2}}{\omega}|\varphi|^{2}
$$

with

$$
c_{0}:=\min _{|\varphi|=1} \int_{V}|\mathcal{F}(\varphi \cdot v)|^{2} d v>0 .
$$

Then Lemma 3.3 is proven.

Now $\nabla \cdot D \nabla$ generates an analytic semigroup and the statements (i) and (ii) follow directly from semigroup theory (see, e.g., Pazy [41]). Since we have no source term in (3.18) statement (iii) is also obvious.

Because of regularity properties of parabolic equations we have the following corollary (see, e.g., Taylor [48]).

Corollary 3.4. For each $m \in \mathbb{N}$ and each $0<\vartheta<\infty$ there is a constant $C_{0}=C_{0}\left(m, \vartheta,\left\|p_{0}(., 0)\right\|_{L^{2}(\tilde{\Omega})}\right)$ such that the solution $p_{0}(\xi, \tau)$ of the parabolic problem (3.18) satisfies

$$
\left\|p_{0}\right\|_{C^{m}\left(\Lambda_{\vartheta}\right)} \leq C_{0}
$$

where $\Lambda_{\vartheta}=\tilde{\Omega} \times(\vartheta, \infty)$.

Remark 3.2. In case of higher regularity of the initial condition, e.g., $p(., v, 0) \in$ $C^{m}(\Omega)$, the initial value of the parabolic problem (3.18) is $C^{m}$ as well. Hence one could argue that the estimate in Corollary 3.4 holds for all $t \in(0, \infty)$. However, since the arguments of $p(\xi, \tau)$ are scaled variables the $C^{m}$-norm scales as $\varepsilon^{-m}$ and the constant $C_{0}$ of this corollary would depend on $\varepsilon>0$. We avoid this scaling by introduction of the time threshold $\vartheta>0$. Starting with general $L^{2}$ initial data for $p_{0}$ the parabolic regularity gives a $C^{m}$-bound independent of $\varepsilon>0$, but for $t>\vartheta$ only. Note that $\vartheta$ is independent of $\varepsilon$ as well.

Remark 3.3. The diffusion equation given by (3.18) cannot lead to aggregation (i.e., nonconstant steady-state solutions) under Neumann boundary conditions even if $D$ depends on $x$ through $\lambda$ (cf. [38]). 
3.2. Structure of the diffusion matrix $\boldsymbol{D}$. Example 3.1 is the simplest case in which the diffusion matrix

$$
D=-\frac{1}{\omega} \int_{V} v \mathcal{F} v d v
$$

is a scalar times the identity, but we can derive more general necessary and sufficient conditions under which this is true. For this we fix $\xi$ and define the momentum per unit mass in the direction $v$ after a jump, assuming a uniform distribution of velocities before the jump, by

$$
\bar{v}(v) \equiv \int T\left(v, v^{\prime}\right) v^{\prime} d v^{\prime} .
$$

For simplicity we later call this the expected velocity. Note the $\bar{v}(v)$ vanishes whenever $T$ is independent of $v^{\prime}$, and that for any $T$

$$
\int_{V} \bar{v}(v) d v=0
$$

i.e., $\bar{v}(v)$ has zero mean. This reflects the fact that the total momentum per unit mass after a jump is zero, since the distribution of velocities before the jump is uniform. For the analysis of isotropy we assume that the set of velocities $V$ is symmetric with respect to $S O(n)$. Since directional changes are described by the distribution function $T$ this assumption is not a restriction. However, if we assume symmetry there is a constant $K_{V}>0$ such that

$$
\int_{V} v v d v=K_{V} I
$$

Especially for fixed speed $V=s S^{n-1}$ we get

$$
K_{V}=\frac{\omega s^{2}}{n} \quad \text { with } \quad \omega=\left|S^{n-1}\right| .
$$

Now consider the following three statements.

(St1). There exists an orthonormal basis $(O N B)\left\{e_{1}, \ldots, e_{n}\right\}$ of $\mathbb{R}^{n}$ such that the coordinate mappings $\phi_{i}: V \rightarrow \mathbb{R}$ given by

$$
\phi_{i}(v)=v_{i}
$$

are eigenfunctions of $\mathcal{L}$ with eigenvalue $\mu \in(-2 \lambda, 0)$ for $1 \leq i \leq n$.

(St2). The expected velocity $\bar{v}(v)$ satisfies

$$
\bar{v}(v) \| v \quad \text { and } \quad \frac{\bar{v}(v) \cdot v}{v^{2}}=\gamma
$$

for all $v \in V$ and a constant $\gamma \in(-1,1)$. We call $\gamma$ the adjoint persistence for reasons that will be clear later.

(St3). There is a constant $d>0$ such that the diffusion matrix has the representation

$$
D=d I
$$


TheOREm 3.5. Assume (T1)-(T4) and assume that $V$ is symmetric with respect to $S O(n)$; then we have

$$
(\mathrm{St} 1) \quad \Longleftrightarrow \quad(\mathrm{St} 2) \quad \Longrightarrow \quad(\mathrm{St} 3) .
$$

The constants $\mu, \gamma$, and $d$ are related as follows. Given $\mu<0$ then

$$
\gamma=\frac{\mu+\lambda}{\lambda}, \quad d=-\frac{K_{V}}{\omega \mu}=\frac{K_{V}}{\omega \lambda(1-\gamma)} .
$$

If $T$ also satisfies the condition (T5), there is a matrix $\mathrm{M}$ such that $\bar{v}(v)=M v$ for all $v \in V$; then all three statements are equivalent.

Proof.

(St1) $\Longleftrightarrow($ St 2$)$. The equivalence of statements 1 and 2 is straightforward:

$(\mathrm{St} 1) \quad \Longleftrightarrow \quad \mathcal{L} v_{i}=\mu v_{i} \quad$ for all $1 \leq i \leq n \quad \Longleftrightarrow \quad-\lambda v_{i}+\lambda(\bar{v}(v))_{i}=\mu v_{i}$,

$$
\Longleftrightarrow(\bar{v}(v))_{i}=\gamma v_{i} \quad \text { with } \quad \gamma=\frac{\mu+\lambda}{\lambda} \Longleftrightarrow \quad \text { (St2). }
$$

(St1) $\Longrightarrow\left(\right.$ St3). Assume (St1). Since $\phi_{i}$ is an eigenfunction of $\mathcal{L}$ and $\phi_{i} \in\langle 1\rangle^{\perp}$ it is also an eigenfunction of $\mathcal{F}$ with eigenvalue $\mu^{-1}$. Then

$$
\begin{aligned}
e_{k} D e_{j} & =-\frac{1}{\omega} \int v_{k} \mathcal{F} v_{j} d v=-\frac{1}{\omega} \int v_{k} v_{j} d v \mu^{-1}=-\frac{1}{\omega \mu} e_{k} \int v v d v e_{j} \\
& =-\frac{K_{V}}{\omega \mu} \delta_{k j} .
\end{aligned}
$$

$(\mathrm{St} 3) \Longrightarrow(\mathrm{St} 1)$ with (T5): Now we assume that (St1) is not valid. Then for any ONB $\left\{e_{1}, \ldots, e_{n}\right\}$ there is an index $1 \leq i \leq n$ such that $\phi_{i}$ is not an eigenfunction of $\mathcal{L}$ with eigenvalue $\mu$. Then either (i) $\phi_{i}$ is not an eigenfunction, or (ii) $\phi_{i}$ is an eigenfunction with eigenvalue $\mu_{i} \neq \mu$.

Consider (i): From condition (T5) it follows that the $n$-dimensional subspace of functions linear in $v$ is invariant under $\mathcal{L}$, hence it is also invariant for $\mathcal{F}$. Then we have a representation $\mathcal{F} \phi_{i}(v)=\alpha \cdot v$ with some $\alpha \in \mathbb{R}^{n}$. Since $\phi_{i}$ is assumed not to be an eigenfunction we have at least one $k \neq i$ with $e_{k} \cdot \alpha \neq 0$. Then

$$
e_{k} D e_{i}=-\frac{1}{\omega} e_{k} \int v \mathcal{F} v_{i} d v=-\frac{1}{\omega} e_{k} \int v \alpha \cdot v d v=-\frac{K_{V}}{\omega} e_{k} \cdot \alpha \neq 0 .
$$

Hence $D$ has nonzero off-diagonal entries, which contradicts (St3).

Consider (ii): If $\phi_{i}$ is an eigenfunction with eigenvalue $\mu_{i} \neq \mu$ a similar calculation shows the contradiction

$$
d=-\frac{K_{V}}{\omega \mu}=e_{i} D e_{i}=-\frac{K_{V}}{\omega \mu_{i}} .
$$

Remark 3.4.

1. If $T$ has the symmetric form

$$
T\left(v, v^{\prime}\right)=t\left(\left|v-v^{\prime}\right|\right),
$$

then the following subspaces of $L^{2}(V)$ are invariant under $\mathcal{L}$ : the constant functions $\langle 1\rangle$, the $v$-linear functions $\langle v\rangle$, and the orthogonal complement $K:=\langle 1, v\rangle^{\perp}$ (see, e.g., 
Alt [1]), and (T5) is satisfied in this case. As we will see in a later example, the spectral problem can easily be solved in this case for two space dimensions, but here we make one further connection with previous work.

For a fixed incoming velocity $v^{\prime}$, the average velocity after reorientation is defined by

$$
\hat{v}=\int T\left(v, v^{\prime}\right) v d v
$$

and the average speed is defined as

$$
\hat{s}=\int T\left(v, v^{\prime}\right)\|v\| d v .
$$

The index of directional persistence, which is defined as

$$
\psi_{d} \equiv \frac{\hat{v} \cdot v^{\prime}}{\hat{s} s^{\prime}},
$$

measures the tendency of the motion to persist in the direction of $v^{\prime}$. This definition provides the basis for calling the parameter $\gamma$ defined earlier the adjoint persistence. If the speed does not change with reorientation and the turning probability depends only on the cone angle $\theta \equiv \arccos \left(\left(v \cdot v^{\prime}\right) / s s^{\prime}\right)$ between $v^{\prime}$ and $v$, then $T\left(v, v^{\prime}\right)$ is replaced by

$$
h\left(\theta\left(v, v^{\prime}\right)\right),
$$

with a suitable change in the measure. Here $h$ is normalized so that

$$
\begin{aligned}
2 \int_{0}^{\pi} h(\theta) d \theta=1 \quad \text { for } \mathrm{n}=2, \\
2 \pi \int_{0}^{\pi} h(\theta) \sin \theta d \theta=1 \quad \text { for } \mathrm{n}=3 .
\end{aligned}
$$

In this case $\psi_{d}$ in (3.21) is independent of $v^{\prime}$ and

$$
\hat{v}=\psi_{d} v^{\prime}
$$

where $\psi_{d}$ is given by

$$
\psi_{d}= \begin{cases}2 \int_{0}^{\pi} h(\theta) \cos \theta d \theta & \text { for } n=2, \\ 2 \pi \int_{0}^{\pi} h(\theta) \cos \theta \sin \theta d \theta & \text { for } n=3 .\end{cases}
$$

Since $T$ is symmetric $\bar{v}=\hat{v}$, the adjoint persistence $\gamma$ is equal to $\psi_{d}$, and the diffusion coefficient is

$$
d=\frac{s^{2}}{n \lambda\left(1-\psi_{d}\right)} .
$$

This result has been derived previously in a variety of ways (cf. $[40,31,1,36]$ ). In general $\gamma$ and $\psi_{d}$ are distinct. 
2. When $D$ is not isotropic there are nonzero off-diagonal elements because some of the coordinate mappings $\phi_{i}$ are not eigenfunctions of $\mathcal{L}$. Examples of this are given in the context of the chemotaxis equations in Part II.

3. We will also see in Part II that (St2) can be used to check the isotropy of the diffusion matrix and hence of the parabolic limit equation.

Example 3.2. Suppose that the kernel $T\left(v, v^{\prime}\right)$ depends only on the cone angle $\theta$ between $v$ and $v^{\prime}$ and consider a kernel with a two-term trigonometric expansion of the form

$$
T\left(v, v^{\prime}\right)=\frac{1}{\omega}(1+a \cos \alpha)
$$

for a suitable function $a=a(x, t)$. We assume constant speed, i.e., $V=s S^{n-1}$, with $s>0$, and one can verify that $\int T\left(v, v^{\prime}\right) d v=1$.

The expected velocity is

$$
\bar{v}(v)=\frac{1}{\omega} \int\left(1+\frac{a}{s^{2}} v \cdot v^{\prime}\right) v^{\prime} d v^{\prime}=\frac{a}{n} v
$$

and therefore the index of persistence is $\gamma=a / n=\psi_{d}$ by virtue of the symmetry of $T$, and to ensure that $|\gamma|<1$ we have to assume that $a<n$. Then Theorem 3.5 applies and $D=d I$ with diffusion parameter

$$
d=\frac{s^{2}}{\lambda(n-a)}
$$

which depends on $(\xi, \tau)$ via $a(x, t)$

Example 3.3. The foregoing analysis can be done completely in $\mathbb{R}^{2}$. Suppose that the speed is constant, and write

$$
v=s(\cos \theta, \sin \theta) .
$$

We write $T\left(v, v^{\prime}\right)=h\left(\theta, \theta^{\prime}\right)$, where $\theta, \theta^{\prime} \in[0,2 \pi]$ and we assume that $h\left(\theta, \theta^{\prime}\right)=$ $h\left(\theta^{\prime}, \theta\right)$. This ensures normality of the turning operator $\mathcal{L}$ and by virtue of Lemma 3.1 we can construct the pseudoinverse $\mathcal{F}$ once we know the spectral decomposition of $\mathcal{L}$. We must first consider the spectral problem

$$
\int T\left(v, v^{\prime}\right) \phi\left(v^{\prime}\right) d v^{\prime}=\int_{0}^{2 \pi} h\left(\theta, \theta^{\prime}\right) \phi\left(\theta^{\prime}\right) d \theta^{\prime}=\hat{\mu} \phi .
$$

We use the orthonormal system $\left\{e^{i n \theta} / \sqrt{2 \pi}\right\}_{n \in \mathbf{Z}}$ and write

$$
h\left(\theta, \theta^{\prime}\right)=\sum_{n, m=-\infty}^{\infty} a_{n m} e^{\imath n \theta} / \sqrt{2 \pi} e^{\imath m \theta^{\prime}} / \sqrt{2 \pi} .
$$

The symmetry condition then implies that $a_{n m}=a_{m n}$. Since we also assume that $h$ is real we must have that $a_{n m}=\bar{a}_{-n,-m}$ or $a_{-n,-m}=\bar{a}_{n, m}$.

We can also expand

$$
\phi(\theta)=\sum_{k=-\infty}^{\infty} b_{k} e^{\imath k \theta} / \sqrt{2 \pi}
$$


and since $\phi$ is real we have $b_{-k}=\bar{b}_{k}$. Therefore (3.29) becomes

$$
\begin{aligned}
\frac{1}{2 \pi} \int_{0}^{2 \pi} \sum_{k, n, m} b_{k} a_{n m} e^{\imath n \theta} e^{\imath m \theta^{\prime}} e^{\imath k \theta^{\prime}} d \theta & =\hat{\mu} \sum_{k} b_{k} e^{\imath k \theta}, \\
\sum_{k, n} b_{k} a_{n k} e^{\imath n \theta} & =\hat{\mu} \sum_{k} b_{k} e^{\imath k \theta}, \\
\sum_{n}(A b)_{n} e^{\imath n \theta} & =\hat{\mu} \sum_{n} b_{n} e^{\imath n \theta}, \\
\sum_{n}(A-\hat{\mu} I) b_{n} e^{\imath n \theta} & =0 .
\end{aligned}
$$

Therefore we have that

$$
[(A-\hat{\mu} I) b]_{n}=0, \quad|n|=0,1,2, \ldots .
$$

We know that $A$ is symmetric and that $a_{-n,-m}=\bar{a}_{n, m}$. One can obtain the eigenvalues and eigenfunctions from this equation, analytically in simple cases and numerically if necessary, and then (3.13) gives the pseudoinverse $\mathcal{F}$.

Example 3.4. We furthermore assume that in the preceding example the kernel depends only on $\theta-\theta^{\prime}$, for then we can assume that

$$
h\left(\theta-\theta^{\prime}\right)=\sum_{n=0}^{\infty} a_{n} \cos n\left(\theta-\theta^{\prime}\right)=\sum_{n=0}^{\infty} a_{n}\left(\cos n \theta \cos n \theta^{\prime}+\sin n \theta \sin n \theta^{\prime}\right) .
$$

We already know that a symmetric kernel gives rise to an isotropic diffusion matrix (Theorem 3.5). However, it turns out to be helpful in understanding the entire approximation process if for one example we use formula (3.16) to explicitly calculate $D$. We may assume that

$$
\phi=\sum_{k=0}^{\infty} c_{k} \cos k \theta+d_{k} \sin k \theta,
$$

and then the eigenvalue problem is

$$
\begin{aligned}
& \hat{\mu} \sum_{k} c_{k} \cos k \theta+d_{k} \sin k \theta \\
& =\int_{0}^{2 \pi} \sum_{n, k} a_{n}\left(c_{k} \cos k \theta^{\prime}+d_{k} \sin k \theta^{\prime}\right)\left(\cos n \theta \cos n \theta^{\prime}+\sin n \theta \sin n \theta^{\prime}\right) d \theta^{\prime} \\
& =\sum_{k} a_{k} c_{k} \cos k \theta \int_{0}^{2 \pi} \cos ^{2} k \theta^{\prime} d \theta^{\prime}+\sum_{k} a_{k} d_{k} \sin k \theta \int_{0}^{2 \pi} \sin ^{2} k \theta^{\prime} d \theta^{\prime} \\
& =2 \pi a_{0}+\pi \sum_{k \geq 1} a_{k}\left(c_{k} \cos k \theta+d_{k} \sin k \theta\right) .
\end{aligned}
$$

Therefore

$$
2 \pi a_{0}-\hat{\mu_{0}}+\sum_{k \geq 1}\left(\pi a_{k}-\hat{\mu}\right)\left(c_{k} \cos k \theta+d_{k} \sin k \theta\right)=0
$$


and this effectively diagonalizes the problem, for now we have

$$
\hat{\mu}_{0}=2 \pi a_{0} \quad \text { and } \quad \hat{\mu}_{k}=\pi a_{k} \quad \text { for } \quad k=1,2, \ldots
$$

and $\left(c_{k}, d_{k}\right)$ chosen by normalization. Thus

$$
\hat{\mu}_{0}=1, \quad \hat{\mu}_{k}=\pi a_{k}, \quad \text { and } \quad \phi_{k}=c_{k} \cos k \theta+d_{k} \sin k \theta .
$$

For $k=1,2, \ldots$ each eigenvalue $\hat{\mu}_{k}$ has a two-dimensional eigenspace which is spanned by

$$
\phi_{k}^{1}(\theta)=\frac{1}{\sqrt{\pi}} \cos k \theta, \quad \phi_{k}^{2}(\theta)=\frac{1}{\sqrt{\pi}} \sin k \theta .
$$

Then the orthogonal projections $P_{k}$ are given by

$$
P_{k} \phi(v)=\int_{0}^{2 \pi} \frac{\cos k \theta^{\prime}}{\sqrt{\pi}} \phi\left(\theta^{\prime}\right) d \theta^{\prime} \frac{\cos k \theta}{\sqrt{\pi}}+\int_{0}^{2 \pi} \frac{\sin k \theta^{\prime}}{\sqrt{\pi}} \phi\left(\theta^{\prime}\right) d \theta^{\prime} \frac{\sin k \theta}{\sqrt{\pi}} .
$$

From Lemma 3.1 we know that $\mathcal{F}$ has a spectral representation. Hence formula (3.16) reads

$$
D=-\frac{s^{2}}{2 \pi} \int_{0}^{2 \pi}\left(\begin{array}{c}
\cos \theta \\
\sin \theta
\end{array}\right) \sum_{j=1}^{\infty} \mu_{j}^{-1} P_{j}(\cos \theta, \sin \theta) d \theta,
$$

where $\mu_{j} \equiv-\lambda\left(1-\hat{\mu}_{j}\right)$. After rearranging we obtain

$$
D=-\frac{s^{2}}{2 \pi} \int_{0}^{2 \pi} \sum_{j=1}^{\infty} \mu_{j}^{-1}\left(\begin{array}{cc}
\cos \theta\left(P_{j} \cos \theta\right) & \cos \theta\left(P_{j} \sin \theta\right) \\
\sin \theta\left(P_{j} \cos \theta\right) & \sin \theta\left(P_{j} \sin \theta\right)
\end{array}\right) d \theta .
$$

Since $P_{j}$ are projections onto the $j$ th Fourier modes we have

$$
P_{j} \cos \theta=\cos \theta \delta_{1, j}, \quad P_{j} \sin \theta=\sin \theta \delta_{1, j} .
$$

Hence we get

$$
\begin{aligned}
D & =-\frac{s^{2}}{2 \pi} \int_{0}^{2 \pi} \mu_{1}^{-1}\left(\begin{array}{cc}
\cos ^{2} \theta & \cos \theta \sin \theta \\
\sin \theta \cos \theta & \sin ^{2} \theta
\end{array}\right) d \theta \\
& =-\frac{s^{2}}{2} \mu_{1}^{-1}\left(\begin{array}{ll}
1 & 0 \\
0 & 1
\end{array}\right)=\frac{s^{2}}{2 \lambda\left(1-\hat{\mu}_{1}\right)} I .
\end{aligned}
$$

It follows from (3.26) that $\hat{\mu}_{1}=\psi_{d}$, and therefore the diffusion coefficient reduces to

$$
d=\frac{s^{2}}{2 \lambda\left(1-\psi_{d}\right)}
$$

as in (3.27).

Remark 3.5 (elliptic scaling). One can also consider the previous space scale and the faster time scale given by $\theta=\varepsilon t$. The transport equation (1.1) reads in this scaling

$$
\varepsilon \frac{\partial p}{\partial \theta}+\varepsilon v \cdot \nabla p=-\lambda p+\lambda \int_{V} T\left(v, v^{\prime}\right) p\left(v^{\prime}\right) d v^{\prime}
$$


As before, we assume an expansion in $\varepsilon$ of the form

$$
p=\sum_{k} \varepsilon^{k} p_{k}
$$

and find that

$$
\begin{array}{ll}
\varepsilon^{0}: & \mathcal{L} p_{0}=0, \\
\varepsilon^{1}: & \frac{\partial p_{0}}{\partial \theta}+v \cdot \nabla p_{0}=\mathcal{L} p_{1} .
\end{array}
$$

It follows that $p_{0}=p_{0}(\theta, \xi)$ and one finds that $p_{0}$ and

$$
p_{1}=\mathcal{F}\left(v \cdot \nabla p_{0}\right)
$$

are independent of $\theta \geq 0$.

The $\mathcal{O}\left(\varepsilon^{2}\right)$ solvability condition leads to the equation

$$
\nabla \cdot D \nabla p_{0}=0
$$

with the diffusion matrix given in (3.16). Hence $p_{0}$ has to solve an elliptic problem (see Lemma 3.3). The same is true at higher orders, and thus the elliptic scaling leads to a stationary problem.

The elliptic scaling (for a symmetric kernel $T$ ) is also used in [16], but there it is claimed that the solvability condition leads to a parabolic limit equation for the lowest-order term in the perturbation expansion, rather than the elliptic equation obtained above. In our notation (and omitting the advection term) (2.42i) in [16] reads

$$
\frac{\partial p_{0}}{\partial \theta}=\nabla \cdot D \nabla p_{0}
$$

where $D=C \varepsilon^{2}$ and $C \sim \mathcal{O}(1)$. In fact, following the arguments in [16] which lead from (2.40) to (2.41), the diffusion constant must be proportional to $\varepsilon$ and not to $\varepsilon^{2}$. In any case the limiting equation obtained from the solvability condition depends on $\varepsilon$, which indicates that the matching is not internally consistent. To obtain a limit parabolic equation which is independent of $\varepsilon$ requires use of the time scaling we defined earlier.

\section{The parabolic limit.}

TheOrem 4.1. Assume (T1)-(T4). For $k \geq 2$ define the sequence of functions $\left(p_{0}(\xi, \tau), p_{1}(\xi, v, \tau), \ldots, p_{k}(\xi, v, \tau)\right)$ by the following conditions:

(a1) $\quad p_{0}$ solves the parabolic initial value problem (3.18),

(a4) $\quad p_{1}(\xi, v, \tau):=\mathcal{F}\left(v \cdot \nabla p_{0}(\xi, \tau)\right)$

(a5) $\quad p_{j}(\xi, v, \tau):=\mathcal{F}\left(p_{j-2, \tau}+v \cdot \nabla p_{j-1}\right)$ for all $2 \leq j \leq k$, 
and $\vartheta, C_{0}$ are the constants from Corollary 3.4. Then

$$
q_{k}:=\sum_{j=0}^{k} \varepsilon^{j} p_{j}
$$

satisfies for each $\vartheta / \varepsilon^{2}<t<\infty$ and each $x \in \Omega$

$$
\left\|p(x, ., t)-q_{k}(x, ., t)\right\|_{L^{2}(V)}^{2} \leq C \varepsilon^{k+1},
$$

where the constant $C$ depends on $\mu_{2}, \omega$ powers of $s$ of highest order $2 k$ and on $C_{0}$.

Proof. The proof is by induction.

Start of induction $k=2$. Let us denote the approximation constructed above by

$$
q_{2}(x, v, t)=p_{0}(\xi, \tau)+\varepsilon p_{1}(\xi, v, \tau)+\varepsilon^{2} p_{2}(\xi, v, \tau)
$$

for $\varepsilon>0$. The properties (a1)-(a5) for $k=2$ have been derived in the previous section.

We assume that $p=q_{2}+\varepsilon^{3} r_{3}$ and show that $r_{3}$ is bounded pointwise in $(\xi, \tau)$ independent of $\varepsilon>0$. In light of condition (3.12) it follows that

$$
\int_{V} r_{3}(\xi, v, \tau) d v=0 .
$$

We introduce $p=q_{2}+\varepsilon^{3} r_{3}$ into the scaled transport equation (3.3) and collect orders of $\varepsilon$. Since $p_{0}, p_{1}$, and $p_{2}$ are solutions of the $\varepsilon^{0}, \varepsilon^{1}$, and $\varepsilon^{2}$ equations (3.5)-(3.7), respectively, it remains to study (3.8) for $i=3$. To apply the pseudoinverse $\mathcal{F}$ we have to fulfill a solvability condition

$$
0=\int_{V} p_{1, \tau}+v \cdot \nabla p_{2} d v
$$

Using (a2) and (a3) this condition is satisfied. We can write the solution of (3.8) with $i=3$ as

$$
\begin{aligned}
r_{3} & =\mathcal{F}\left(p_{1, \tau}+v \cdot \nabla p_{2}\right) \\
& =\mathcal{F}\left(\mathcal{F}\left(v \cdot \nabla p_{0, \tau}\right)+v \cdot \nabla \mathcal{F}\left\{p_{0, \tau}+v \cdot \nabla \mathcal{F}\left(v \cdot \nabla p_{0}\right)\right\}\right) .
\end{aligned}
$$

Now we estimate $r_{3}$ in $L^{2}(V)$ :

$$
\begin{aligned}
\left\|r_{3}(\xi, ., \tau)\right\|_{L^{2}(V)}^{2} \leq & \frac{2}{\mu_{2}^{2}}\left(\left\|\mathcal{F}\left(v \cdot \nabla p_{0, \tau}\right)\right\|_{L^{2}(V)}^{2}\right. \\
& \left.+\left\|v \cdot \nabla \mathcal{F}\left(p_{0, \tau}+v \cdot \nabla \mathcal{F}\left(v \cdot \nabla p_{0}\right)\right)\right\|_{L^{2}(V)}^{2}\right) .
\end{aligned}
$$

Using Corollary 3.4 we estimate the first term in the brackets of the right-hand side of (4.2) in detail.

$$
\begin{aligned}
\left\|\mathcal{F}\left(v \cdot \nabla p_{0, \tau}\right)\right\|_{L^{2}(V)}^{2} & \leq \frac{1}{\mu_{2}^{2}}\left\|v \cdot \nabla p_{0, \tau}(\xi, v, \tau)\right\|_{L^{2}(V)}^{2} \\
& \leq \frac{s^{2}}{\mu_{2}^{2}}\|\| p_{0, \tau}(\xi, v, \tau)\left\|_{C^{1}(\tilde{\Omega})}\right\|_{L^{2}(V)}^{2} \\
& \leq \frac{s^{2}}{\mu_{2}^{2}} \omega^{2} C_{0}^{2}
\end{aligned}
$$


for $\vartheta<\tau<\infty$. A similar estimate for the second term in (4.2) leads with the use of Corollary 3.4 to

$$
\left\|v \cdot \nabla \mathcal{F}\left(p_{0, \tau}+v \cdot \nabla \mathcal{F}\left(v \cdot \nabla p_{0}\right)\right)\right\|_{L^{2}(V)}^{2} \leq \frac{2 s^{2}}{\mu_{2}^{2}} \omega^{2} C_{0}^{2}\left(1+\frac{s^{4}}{\mu_{2}^{2}}\right)
$$

for $\vartheta<\tau<\infty$. Hence, all together we have

$$
\left\|r_{3}(\xi, ., \tau)\right\|_{L^{2}(V)}^{2} \leq \frac{2 s^{2} \omega^{2} C_{0}^{2}}{\mu_{2}^{4}}\left(3+\frac{2 s^{4}}{\mu_{2}^{2}}\right)=: C^{2}
$$

for $\vartheta<\tau<\infty$ and all $\xi \in \tilde{\Omega}$. Since $\tau=\varepsilon^{2} t$ it follows that for all $x \in \Omega$

$$
\left\|r_{3}(x, ., t)\right\|_{L^{2}(V)} \leq C \text { for all } \frac{\vartheta}{\varepsilon^{2}}<t<\infty .
$$

Here $C=C\left(C_{0}, s, \mu_{2}\right)$.

Induction $l \rightarrow l+1$. Assume the statement of Theorem 4.1 is true for all $j<$ $l+1 \leq k$ and assume (a1)-(a5) for $l+1$. We consider

$$
q_{l+1}=\sum_{j=0}^{l+1} \varepsilon^{j} p_{j}
$$

and define $r_{l+2}(\xi, v, \tau)$ by

$$
p=q_{l+1}+\varepsilon^{l+2} r_{l+2} .
$$

We show that $r_{l+2}$ is bounded in $L^{2}(V)$, pointwise in $(\xi, \tau)$, independent of $\varepsilon>0$. We use the scaled transport equation (3.3) and collect terms of order $\varepsilon^{l+2}$ :

$$
\mathcal{L} r_{l+2}=p_{l, \tau}+v \cdot \nabla p_{l+1}
$$

From ( $a 2)$ and (a3) it directly follows that this equation is solvable, hence

$$
r_{l+2}=\mathcal{F}\left(p_{l, \tau}+v \cdot \nabla p_{l+1}\right) .
$$

We estimate $r_{l+2}$ using property $(2.6)$ of $\mathcal{F}$ :

$$
\left\|r_{l+2}(\xi, ., \tau)\right\|_{L^{2}(V)}^{2} \leq \frac{1}{\mu_{2}^{2}}\left(\left\|p_{l, \tau}(\xi, ., \tau)\right\|_{L^{2}(V)}^{2}+\left\|v \cdot \nabla p_{l+1}(\xi, ., \tau)\right\|_{L^{2}(V)}^{2}\right) .
$$

Lemma 4.2. For each $2 \leq j \leq l+1$ there is a constant $C_{j}>0$ such that for each $(\tau, \xi) \in \Lambda_{\vartheta}$ and for each derivative $D^{\alpha}$ with multi-index $\alpha \in \mathbb{N}^{n+1}$, with respect to the partial derivatives $\left\{\frac{\partial}{\partial t}, \frac{\partial}{\partial x_{1}}, \ldots, \frac{\partial}{\partial x_{n}}\right\}$ we have

$$
\left\|D^{\alpha} p_{j}\right\|_{L^{2}(V)}^{2} \leq C_{j}\left\|p_{0}\right\|_{C^{j+|\alpha|}\left(\Lambda_{\vartheta}\right)}^{2} .
$$

The constant $C_{j}$ depends on $\mu_{2}, \omega$, on powers of $s$ of highest order $2 j$ and of lower order norms of $p_{0}$. 
Proof. The proof is by induction.

Start of induction: $j=2$. Let $\mathcal{D}^{2}$ denote the total second derivative with respect to space.

$$
\begin{aligned}
\left\|D^{\alpha} p_{2}\right\|_{L^{2}(V)}^{2} & =\left\|\mathcal{F}\left(D^{\alpha} p_{0, \tau}+v \cdot \nabla D^{\alpha} p_{1}\right)\right\|_{L^{2}(V)}^{2} \\
& \leq \frac{1}{\mu_{2}^{2}}\left(\left\|D^{\alpha} p_{0, \tau}\right\|_{L^{2}(V)}^{2}+s^{2}\left\|\nabla \mathcal{F}\left(v \cdot \nabla D^{\alpha} p_{0}\right)\right\|_{L^{2}(V)}^{2}\right) \\
& \leq \frac{1}{\mu_{2}^{2}} \omega^{2}\left\|D^{\alpha} p_{0}\right\|_{C^{1}\left(\Lambda_{\vartheta}\right)}+\frac{s^{4} \omega^{2}}{\mu_{2}^{4}}\left|D^{\alpha} \mathcal{D}^{2} p_{0}\right| \\
& \leq C_{2}\left\|p_{0}\right\|_{C^{2+|\alpha|}\left(\Lambda_{\vartheta}\right)},
\end{aligned}
$$

where $C_{2}$ depends on $s^{4}, \omega^{2}$ and on $\mu_{2}^{-4}$.

Induction $j-1 \rightarrow j$. We assume that the statement of the above lemma is true for $2 \leq i \leq j-1$.

$$
\begin{aligned}
\left\|D^{\alpha} p_{j}\right\|_{L^{2}(V)}^{2} & =\left\|\mathcal{F}\left(D^{\alpha} p_{j-2, \tau}+v \cdot \nabla D^{\alpha} p_{j-1}\right)\right\|_{L^{2}(V)}^{2} \\
& \leq \frac{1}{\mu_{2}^{2}}\left(\left\|D^{\alpha} p_{j-2, \tau}\right\|_{L^{2}(V)}^{2}+\left\|v \cdot \nabla D^{\alpha} p_{j-1}\right\|_{L^{2}(V)}^{2}\right) \\
& \leq \frac{1}{\mu_{2}^{2}}\left(C_{j-2}\left\|p_{0}\right\|_{C^{j-2+|\alpha|+1}\left(\Lambda_{\vartheta}\right)}^{2}+s^{2} C_{j-1}\left\|p_{0}\right\|_{C^{j-1+|\alpha|+1}\left(\Lambda_{\vartheta}\right)}^{2}\right) \\
& \leq C_{j}\left\|p_{0}\right\|_{C^{j+|\alpha|}\left(\Lambda_{\vartheta}\right)}^{2},
\end{aligned}
$$

where $C_{j}$ depends on $\mu_{2}, C_{j-1}, C_{j-2}$, on powers of $s$ of maximal order $2 j$, and on $\left\|p_{0}\right\|_{C^{j+|\alpha|-1}\left(\Lambda_{\vartheta}\right)}$.

Finally, we estimate the residuum $r_{l+2}$ : with use of Corollary 3.4 and Lemma 4.2:

$$
\begin{aligned}
\left\|r_{l+2}\right\|_{L^{2}(V)}^{2} & \leq \frac{1}{\mu_{2}^{2}}\left(C_{l}\left\|p_{0}\right\|_{C^{l+1}\left(\Lambda_{\vartheta}\right)}^{2}+s^{2} C_{l+1}\left\|p_{0}\right\|_{C^{l+2}\left(\Lambda_{\vartheta}\right)}^{2}\right) \\
& \leq \tilde{C}\left\|p_{0}\right\|_{C^{l+2}\left(\Lambda_{\vartheta}\right)} \\
& \leq \tilde{C} C_{0} .
\end{aligned}
$$

Note that the constant $C=\tilde{C} C_{0}$ does not depend on $\varepsilon>0$.

Remark 4.1. Note that in general $p_{k}$ for $k \geq 1$ are unique only up to a function constant in $v$. However, in (3.12) and in (a2) we stipulated that all mass is carried by $p_{0}$. Hence this constant component is zero. If we did not make assumption (3.12), then for each order of $\varepsilon$ a parabolic equation would appear. Its solution would give the $v$-constant part of $p_{k}$ (see Alt [2] in the case of a symmetric kernel).

Remark 4.2 (parabolic limit). Note that there is another interpretation of this result. Consider the linear transport equation with turning rates of order $\lambda=O\left(\varepsilon^{-2}\right)$ and speeds of order $s=O\left(\varepsilon^{-1}\right)$. Then we introduce $\tilde{s}=\varepsilon s$ and $\tilde{\lambda}=\varepsilon^{2} \lambda$ and again we obtain the scaled transport equation (3.3) with $\tilde{s}, \tilde{\lambda}$ instead of $s, \lambda$. Then Theorem 4.1 means that for $\varepsilon \rightarrow 0$ the transport equation converges to the parabolic limit (3.18). The solution $p_{0}$ of the parabolic limit alone will lead to an approximation that is $\mathcal{O}(\varepsilon)$. Here we also use $p_{1}$ and $p_{2}$ to get an approximation that is $\mathcal{O}\left(\varepsilon^{3}\right)$.

5. Discussion. The reader who peruses some of the foregoing literature will be struck by the plethora of assumptions and the complexity of the analysis that leads to the diffusion approximation. We believe that our analysis is significantly more 
transparent than many previous analyses, and we hope that the reader is convinced that there is no mystery behind the diffusion approximation; it can be obtained by a rather straightforward analysis based on regular perturbation techniques. There are some essential hypotheses on the turning operator that are necessary in order to obtain a diffusion approximation, and our derivation provides a clearer insight as to what a minimal set of hypotheses must contain. While there is intrinsic merit to a simplified derivation of equations as important as the diffusion equation derived here or the chemotaxis equations derived in Part II, perhaps more important is that a clear derivation lays bare the essentials and provides the basis for a more direct interpretation of the diffusion tensor and chemotactic sensitivity (defined in Part II) in terms of more fundamental characteristics of the motion.

Two major facts have emerged from the analysis done here. First, the parabolic limit of the velocity-jump process (3.18) is anisotropic in general, as has been previously observed by Papanicolaou [39] for the backward equation and in several papers for the forward chemotaxis transport model, namely, as a special case in Alt [1] and in Dickinson and Tranquillo [7]. The results of Papanicolaou [39] are closest to those obtained here, and for comparison we will denote any formula and abbreviation from that paper with brackets \{\} . His conditions on $\{$ p. 350$\}$ on the $\{$ "frozen collision process" $\}$ correspond to our conditions on the operator $\mathcal{L}$ and $\mathcal{T}$. His condition $\{(6.6)\}$ corresponds to our $\int T\left(v, v^{\prime}\right) d v=1$. The definition of $\left\{\mu_{0}\right\}$ in $\{(6.7)\}$ has the character of a pseudoinverse $\mathcal{F}$, since $\left\{P_{0}\right\}$ is a solution of a backward equation $\{(6.4)\}$, which defines (roughly) an inverse. However the direct correspondence to our $\mathcal{F}$ is not obvious. Condition $\{(6.8)\}$ seems to be similar to our spectral gap condition $\left\|\mathcal{T}^{*}\right\|_{\langle 1\rangle \perp}<1$. The $\{$ "centering" $\}$ condition on the field $\{F\}$, mentioned on $\{\mathrm{p}$. 340 , top line $\}$, or on $\{$ p. 350 , bottom line $\}$ corresponds to our symmetry condition $\int v d v=0$, and the diffusion parameters in $\{(6.13)\}$ define a matrix analogous to our $D$. The velocity-jump process studied here is obtained from the jump process in [39] by choosing the external force field $H=0$, which means that the velocity is constant between jumps, and hence the results in [39] apply to the backward equation corresponding to (1.1) for a one-particle random walk. On the other hand, there are several differences. Here we develop a method to generate higher order approximations, whereas in [39] only the first-order approximation is considered. Moreover, the notion of the pseudoinverse $\mathcal{F}$ gives a clearer insight into the dependencies of parameters like the diffusion coefficient and chemotactic sensitivity (if applied to chemotaxis) on the model parameters $\lambda$ and T. In [39] there is a persistent force $H$ that could incorporate imposed biases, whereas in Part II we systematically classify chemotactic biases that arise in $\lambda$ and $T$ from dependence on external fields. Finally, we have derived necessary and sufficient conditions for isotropy of the limiting equation.

Many previous analyses, including ours, apply only when there is no direct interaction among particles, although they may interact via an external signal. The rigorous derivation of transport equations as (1.1) or even of diffusion equations from stochastic processes of interacting populations is currently an active area of research (see, e.g., Durett and Neuhauser [11], Stevens [44], or Morale and Capasso [33]). As will be discussed further in Part II, some of these derivations lead to equations identical to those based on the assumption of no interactions, but this apparent contradiction remains to be resolved.

In Part II we investigate how the classical PKSA chemotaxis equation, which describes the population-level response to external chemical signals, can be obtained from a microscopic description of run-length and turning behavior in response to these 
signals. The simplest form of the PKSA equation, coupled with an evolution equation for the external signal, is

$$
\begin{aligned}
& p_{t}=\nabla(d \nabla p-\chi(S) p \nabla S), \\
& S_{t}=A \Delta S+f(p, S) .
\end{aligned}
$$

Here $p(x, t)$ denotes the population density and $S(x, t)$ denotes the density of the external signal. In Part II it is shown that the classical PKSA chemotaxis equation emerges in a very restricted limit, namely, when the bias term is precisely of $\mathcal{O}(\varepsilon)$ in the scaling used here. If the bias is too large it either modifies the diffusion tensor or may preclude a diffusion approximation at all. In Part II we study turning rates and turning kernels of the form

$$
\begin{aligned}
T\left(v, v^{\prime}, S(\cdot)\right) & =T_{0}\left(v, v^{\prime}\right)+\varepsilon^{k} T_{1}\left(v, v^{\prime}, S(\cdot)\right), \quad k=0,1,2, \ldots, \\
\lambda(v, S(\cdot)) & =\lambda_{0}+\varepsilon^{l} \lambda_{1}(v, S(\cdot)), \quad l=1,2, \ldots
\end{aligned}
$$

As an example, we briefly describe an application to the slime mold $D d$, where the perturbations have the form $\lambda=\lambda_{0}+\varepsilon \lambda_{1}$ and $T=T_{0}+\varepsilon T_{1}$. Then the parabolic limit equation is

$$
\frac{\partial p_{0}}{\partial \tau}=\nabla\left(D \nabla p_{0}-u_{c} p_{0}\right)
$$

with diffusion tensor $D$, defined via the pseudoinverse $\mathcal{F}_{0}$ of the unperturbed operator turning operator, given by

$$
D=-\frac{1}{\omega} \int v \mathcal{F}_{0} v d v
$$

In addition, the chemotactic velocity is given by

$$
u_{c}=-\frac{\lambda_{0}}{\omega} \int v \mathcal{F}_{0} \beta_{1}(v) d v-\frac{1}{\omega} \int v \mathcal{F}_{0}\left(\lambda_{1}(v, S(\cdot))-\bar{\lambda}_{1}(v, S(\cdot))\right) d v
$$

wherein

$$
\beta_{1}(v)=\int T_{1}\left(v, v^{\prime}\right) d v^{\prime} \quad \text { and } \quad \bar{\lambda}_{1}(v)=\int \lambda_{1}\left(v^{\prime},\right) T_{0}\left(v, v^{\prime}\right) d v^{\prime} .
$$

If $T_{0}$ is symmetric then $\mathcal{F}_{0}$ is multiplication with a scalar. If moreover the perturbations $T_{1}$ and $\lambda_{1}$ are linear in $\nabla S$, then the resulting equation is the classical PKSA equation.

\section{REFERENCES}

[1] W. ALt, Biased random walk models for chemotaxis and related diffusion approximations, J. Math. Biol., 9 (1980), pp. 147-177.

[2] W. Alt, Singular perturbation of differential integral equations describing biased random walks, J. Reine Angew. Math., 322 (1981), pp. 15-41.

[3] R. Beals and V. Protopopescu, Abstract time dependent transport equations, J. Math. Anal. Appl., 121 (1987), pp. 370-405.

[4] C. Cercignani, R. Illner, and M. Pulvirenti, The Mathematical Theory of Diluted Gases, Springer, New York, 1994.

[5] S. Chapman and T. G. Cowling, The Mathematical Theory of Non-Uniform Cases, Cambridge University Press, Cambridge, 1964. 
[6] J. B. Conway, A Course in Functional Analysis, Springer, New York, 1985.

[7] R. B. Dickinson and R. T. Tranquillo, Transport equations and indices for random and biased cell migration based on single cell properties, SIAM J. Appl. Math., 55 (1995), pp. 1419-1454.

[8] S.R. Dunbar, A branching random evolution and a nonlinear hyperbolic equation, SIAM J. Appl. Math., 48 (1988), pp. 1510-1526.

[9] S. Dunbar and H. G. Othmer, On a nonlinear hyperbolic equation describing transmission lines, cell movement and branching random walks, in Nonlinear Oscillations in Biology and Chemistry, H. G. Othmer, ed., Lecture Notes in Biomath. 66, Springer, New York, 1986, pp. 274-289.

[10] N. Dunford And J. Schwarz, Linear Operators, Part II: General Theory, Wiley-Interscience, New York, 1964.

[11] R. Durett And C. Neuhauser, Particle systems and reaction-diffusion equations, Ann. Probab., 22 (1994), pp. 289-333.

[12] R. S. ElLIS, Chapman-Enskog-Hilbert expansion for a Markovian model of the Boltzmann equation, Comm. Pure Appl. Math., 26 (1973), pp. 327-359.

[13] P. R. Fisher, R. MerkL, AND G. Gerisch, Quantitative analysis of cell motility and chemotaxis in Dictyostelium discoideum by using an image processing system and a novel chemotaxis chamber providing stationary chemical gradients, J. Cell Biol., 108 (1989), pp. 973984.

[14] R. FÜRTH, Die Brownsche Bewegung bei Berücksichtigung einer Persistenz der Bewegungsrichtung, Z. Phys., 2 (1920), pp. 244-256.

[15] S. Goldstein, On diffusion by discontinuous movements, and on the telegraph equation, Quart. J. Mech. Appl. Math., VI (1951), pp. 129-156.

[16] D. Grunbaum, Advection-diffusion equations for generalized tactic searching behaviors, J. Math. Biol., 38 (1999), pp. 169-194.

[17] G. J. HabetLer AND MATKOWsky, Uniform asymptotic expansion in transport theory with small mean free paths, and the diffusion approximation, J. Math. Phys., 4 (1975), pp. 846854.

[18] K. P. HAdeler, Random walk systems and reaction telegraph equations, in Dynamical Systems and Their Applications in Science, S. v. Strien and S. V. Lunel, eds., Royal Netherlands Academy of Arts and Sciences, Amsterdam, 1995.

[19] K. P. HAdeler, Reaction transport systems, in Mathematics Inspired by Biology, V. Capasso and O. Diekman, eds., CIME Lectures, Florence, 1997.

[20] K. P. HAdeler, Reaction transport equations in biological modeling, Math. Comp. Model., 31 (1998), pp. $75-81$.

[21] P. R. Halmos and V. S. Sunder, Bounded Imtegral Operators on $L^{2}$ Spaces, Springer, Berlin, 1978.

[22] R. Hersh, Random evolutions: A survey of results and problems, Rocky Mountain J. Math., 4 (1974), pp. 443-477.

[23] T. Hillen, A Turing model with correlated random walk, J. Math. Biol., 35 (1996), pp. 49-72.

[24] T. Hillen, Invariance principles for hyperbolic random walk systems, J. Math. Anal. Appl., 210 (1997), pp. 360-374.

[25] T. Hillen, Qualitative analysis of semilinear Cattaneo systems, Math. Models Methods Appl. Sci., 8 (1998), pp. 507-519.

[26] T. Hillen and A. Stevens, Hyperbolic models for chemotaxis in 1-D, Nonlinear Anal., 2000. to appear.

[27] E. E. Holmes, Are diffusion models too simple? A comparison with telegraph models of invasion, Amer. Naturalist, 142 (1993), pp. 779-795.

[28] M. KAC, A stochastic model related to the telegrapher's equation, Rocky Mountain J. Math., 3 (1974), pp. 497-509.

[29] M. A. M. A. Krasnosel'skit, Positive Solutions of Operator Equations, P. Noordhoff, Groningen, The Netherlands, 1964. English translation of Russian original "Polozhitelnye resheniia operatornykh uravnenii."

[30] E. W. LARSEN AND J. B. KelleR, Asymptotic solution of neutron transport problems for small free mean paths, J. Math. Phys., 15 (1974), pp. 75-81.

[31] P. S. Lovely and F. W. Dahlquist, Statistical measures of bacterial motility and chemotaxis, J. Theoret. Biol., 50 (1975), pp. 477-496.

[32] H. MCKean, Chapman-Enskog-Hilbert expansions for a class of solutions of the telegraph equation, J. Math. Phys., 75 (1967), pp. 1-10.

[33] D. Morale, V. Capasso, And K. Oelschläger, On the derivation of the mean-field nonlinear integro-differential equation for a population of aggregating individuals subject to stochastic 
fluctuations, SFB 359, Preprint, 98-39 (1998).

[34] J. Müller AND T. Hillen, Modulation equations and parabolic limits of reaction random walk systems, Math. Methods Appl. Sci., 21 (1998), pp. 1207-1226.

[35] H. G. ОтнмеR, On the significance of finite propagation speeds in multicomponent reacting systems, J. Chem. Phys., 64 (1976), pp. 460-470.

[36] H. G. Othmer, S. R. Dunbar, and W. Alt, Models of dispersal in biological systems, J. Math. Biol., 26 (1988), pp. 263-298.

[37] H. G. Отhmer and T. Hillen, The Diffusion Limit of Transport Equations, Part II: Chemotaxis Equations, manuscript.

[38] H. G. Othmer And A. Stevens, Aggregation, blowup and collapse: The ABCs of taxis in reinforced random walks, SIAM J. Appl. Math., 57 (1997), pp. 1044-1081.

[39] G. C. Papanicolaou, Asymptotic analysis of transport processes, Bull. Amer. Math. Soc. (N.S.), 81 (1975), pp. 330-392.

[40] C. S. Patlak, Random walk with persistence and external bias, Bull. Math. Biophys., 15 (1953), pp. 311-338.

[41] A. PAZY, Semigroups of Linear Operators and Applications to Partial Differential Equations, Springer, New York, 1983.

[42] G. Schneider AND J. MÜller, Bifurcations analysis for a spatially extended random walk system in mathematical biology, Comm. Appl. Anal., in press.

[43] L. A. Segel, Mathematical models for cellular behavior, in Studies in Mathematical Biology, S. A. Levin, ed., Mathematical Association of America, Washington, D.C, 1978, pp. 156190.

[44] A. Stevens, Derivation of chemotaxis equations of moderately interacting stochastic many particle systems, SIAM J. Appl. Math., 61 (2000), pp. 183-212.

[45] D. W. Stroock, Some stochastic processes which arise from a model of the motion of a bacterium, Probab. Theory Related Fields, 28 (1974), pp. 305-315.

[46] A. E. Taylor and D. C. Lay, Introduction to Functional Analysis, John Wiley and Sons, New York, 1980.

[47] G. I. TAYlOR, Diffusion by discontinuous movements, Proc. London Math. Soc. 3, 20 (1921/1922), pp. 196-212.

[48] M. E. TAYlor, Partial Differential Equations III, Springer, New York, 1996. 